\title{
The Quality and in Vitro Efficacy of Amoxicillin/Clavulanic Acid Formulations in the Central Region of Ghana
}

\author{
Henry Nettey, Grace Lovia Allotey-Babington, Philip Debrah, Ofosua Adi-Dako, Manal Shaick, \\ Isaac Kintoh, Francis Arnansi, Makafui Nyagblordzro, Marvin Holison \\ Department of Pharmaceutics and Microbiology, School of Pharmacy, University of Ghana, Legon, Ghana. \\ Email: hnettey@msn.com \\ Received November $11^{\text {th }}, 2013$; revised December $25^{\text {th }}$, 2013; accepted January $6^{\text {th }}, 2014$ \\ Copyright (c) 2014 Henry Nettey et al. This is an open access article distributed under the Creative Commons Attribution License, \\ which permits unrestricted use, distribution, and reproduction in any medium, provided the original work is properly cited. In accor- \\ dance of the Creative Commons Attribution License all Copyrights (c) 2014 are reserved for SCIRP and the owner of the intellectual \\ property Henry Nettey et al. All Copyright (C) 2014 are guarded by law and by SCIRP as a guardian.
}

\section{ABSTRACT}

Aim: To assess the quality and in vitro efficacy of five brands of amoxicillin/clavulanic acid tablet, suspension and injectable preparations selected from pharmacies in the Central Region of Ghana. Method: Using a Stratified Representation Sampling method, forty preparations (tablets, suspensions and injectable powders) containing amoxicillin and clavulanic acid were sampled from nine different locations within the Central Region of Ghana. To determine drug quality, several procedures, namely, content assay, disintegration and dissolution testing were employed. In vitro drug efficacy was determined by comparing the Minimum Inhibitory Concentrations (MIC's) obtained with published values. Results: All tablets passed the disintegration test, with disintegration time ranging between six (6) and fifteen (15) minutes. Analyses of all the tablets for drug content showed $100 \%$ failure (14 out of 14$)$ for amoxicillin and $14 \%$ failure (2 out of 14$)$ for clavulanic acid. Injectable formulations showed similar results. All four (4) samples analyzed for content failed the amoxicillin content assay (0 out of 4) but all passed clavulanic acid assay (4 out of 4). For tablet dissolution tests, there was a $93 \%$ (13 out of 14) pass rate for both amoxicillin and clavulanic acid. Content analysis of all suspension formulations involved twenty-two (22) samples from five (5) brands. Only $41 \%$ (9 out of 22) passed for both amoxicillin and clavulanic acid. All the other samples failed for either amoxicillin, clavulanic acid or both. Results obtained from drug quality tests were confirmed by in vitro efficacy tests against selected microorganisms. Conclusion: The samples were therefore not of good quality, since content assay is the most crucial test. It is hypothesized that this is due to poor storage conditions, and recommendations, such as air conditioning and more structured procedures along the supply chain, are put forward to counteract this.

\section{KEYWORDS}

Amoxicillin; Clavulanic Acid; Minimum Inhibitory Concentration; Dissolution

\section{Introduction}

Amoxicillin belongs to the class of medications called penicillin-like antibiotics. The penicillins belong to the beta-lactam group of antibiotics [1], which are the dominant class of agents currently used for the chemotherapy of bacterial infections worldwide. Amoxicillin acts by inhibiting the synthesis of bacterial cell walls [2]. It inhibits cross-linkage between the linear peptidoglycan polymer chains that make up a major component of the cell wall of Gram-positive bacteria [3]. It is usually the drug of choice within the class because it is better absorbed following oral administration and it is resistant to gastric acid [4]. Amoxicillin is susceptible to degradation by beta-lactamase-producing bacteria, and so may be given with Clavulanic acid to decrease its susceptibility [5]. Clavulanic acid, a $\beta$-lactamase inhibitor [6], is added to amoxicillin to inhibit $\beta$-lactamase and increase the antibacterial effect of amoxicillin. It is however a less potent antibacterial agent as compared to amoxicillin [6]. Clavulanic acid has a similar structure to the beta-lactam antibiotics but binds irreversibly to the beta-lactamase 
enzymes [7]. Amoxicillin and Clavulanic acid combinations are available in oral solid dosage form, powder for reconstitution as suspension and injectable. These two drugs act synergistically to produce the desired therapeutic effect. Their potency depends on content of the active moiety in these dosage forms. This combination is used as a broad spectrum antibiotic for the treatment of a wide range of bacterial infections, including upper and lower respiratory tract infections and infections of the skin and soft tissue structures [5].

One of the World Health Organization's (WHO's) main priorities is the quality of pharmaceuticals. Article 2 of the WHO Constitution states one of the organization's functions as to “develop, establish and promote international standards with respect to food, biological, pharmaceutical and similar products" [8].

The quality of pharmaceuticals is of importance not just on the global scale, but within nations as well. With the high demand for medication in Ghana, and the high amount of revenue generated by the industry, counterfeit drugs have begun to penetrate the Ghanaian market. In February 2013, the Food and Drugs Authority (FDA) in Ghana reported the importation of fake amoxicillin/clavulanic acid tablets into Ghana [9]. Rox-Clav 625 tablets available on the market were found to contain no clavulanic acid, thus making it counterfeit. This is particularly dangerous because without clavulanic acid, the product is highly inefficient.

Unfortunately, administering counterfeit drugs could result in therapeutic failure, toxicity, allergic reactions due to their content, drug resistance, prolonged illness, high cost of treatment and even mortality. Furthermore, other secondary implications are that the companies, pharmacies and distributors involved may lose their credibility, and a lot of financial resources may be lost in investigations and court cases. One should be aware that low quality medicines may not always be the result of problems at the manufacturing stage. They could be because of problems with packaging, transportation, storage conditions and the distribution system.

Whatever the case, low quality medicines are certainly not desirable on the market. Pharmacists, regulatory officials and health inspectors have to be vigilant and perform quality assurance tests on medications available locally. This gives the public confidence in available medications, and helps to take substandard medications out of the system. Increased quality assurance also prevents counterfeit drugs from penetrating the market: for fear that they will be detected.

Quality assurance is generally conducted in the more prominent areas, such as the Greater Accra Region in Ghana. This research project looks at amoxicillin/clavulanic acid formulations in the Central Region of Ghana, and evaluates their quality and efficacy. With the possi- bility of counterfeit drugs on the market, it is important that the pharmaceutical industry cultivates a culture of regular, quality assurance and efficient reporting processes. It is hoped that this research will contribute towards this practice.

\section{Materials and Methods}

\subsection{Sampling Method}

Five (5) brands of amoxicillin/clavulanic acid formulations available in nine (9) districts in the Central region were purchased. The nine (9) districts were Kasoa $(k)$, Agona Swedru (Ag), Apam (Ap), Winneba (W), Mankessim (M), Elmina (E), TwifuPraso (T), Assin Fosu (As) and Cape Coast (C). The five (5) brands were coded A, B, C, D and E. The samples were coded with the brand code first followed by the dosage form, the town of purchase, and then a number subscript indicating the sample number. For example, if two tablet samples of the brand A were purchased from two different pharmacies in Kasoa, the codes will be AT- $\mathrm{K}_{1}$ and AT- $\mathrm{K}_{2}$. In purchasing the samples, a convenience sampling method was used to select which districts to sample from. Convenience sampling involves drawing the sample from that part of the population which is close to hand. The central region is divided into seventeen districts. In employing the method, the 9 districts were selected because they had the highest number of pharmacies/hospitals as recorded by the Pharmacy Council of Ghana [10]. After selecting the 9 districts, several pharmacies that were close at hand were visited in the areas in order to find out which brands of amoxicillin/clavulanic acid were most popularly used. The 5 most popular brands, named above, were thus selected. Then, tablets, powder for suspension and injectables of these brands were purchased from more than one pharmacy in each district. In order to keep records and information about each product, the pharmacist at each pharmacy from which the products were purchased filled a questionnaire to ensure easy traceability of the product.

\subsection{Disintegration Test}

The disintegration test determines the ability of a tablet or capsule to disintegrate within a given period of time when placed in a liquid medium under established experimental conditions. The end of the test is detected when there is no residue of the tablet or capsule on the mesh of the test apparatus aside from insoluble fragments of the tablet coating or the capsule gel [11]. The apparatus consists of a two basket-rack arrangement of six open ended transparent tubes held upright by two plastic plates with six holes equally spaced from each other. A woven wire mesh made of stainless steel having a square weave with holes is attached to the lower plate and two $1000 \mathrm{ml}$ 
beakers are placed in a rectangular shaped glass water tank. A thermostat is used to control the temperature of the liquid medium between $35^{\circ} \mathrm{C}$ and $39^{\circ} \mathrm{C}$, and a control knob for raising and lowering the basket into the beaker of immersion fluid, distilled water, at a constant frequency rate ranging between 29 to 39 cycles per minute.

\subsection{Experimental Procedure}

$750 \mathrm{ml}$ of distilled water was accurately measured using a $1000 \mathrm{ml}$ measuring cylinder and transferred into each of the $1000 \mathrm{ml}$ beakers. Then the disintegration apparatus, QC-21 Disintegration Test System (Hanson Research, California, USA), was calibrated to a temperature of $37^{\circ} \mathrm{C}+/-0.5^{\circ} \mathrm{C}$. To ensure that the temperature was accurate, a glass thermometer was placed in each beaker. Six tablets or capsules of each formulation of amoxicillin/clavulanic acid from a particular area in the central region were individually weighed and each placed in a transparent tube of the first basket rack. To the transparent tubes of the second basket rack, another set of six tablets/capsules was added. The two racks were gently immersed simultaneously into the liquid medium and the run started. This procedure was repeated for all tablet/capsule samples obtained.

\subsection{Amoxicillin and Clavulanic Acid Standard Preparation}

All dissolution and HPLC analysis were done at the Food and Drug Authority (FDA) laboratories in Accra. Amoxicillin Trihydrate RS, lot number P500127 and purity of 85.7\% w/v was obtained from Sigma-Aldrich. Clavulanate Lithium USP, Lot number JOG109 and purity 95.7\%, w/v was obtained as a gift from the (FDA), Accra, Ghana. An approximate amount of $0.025 \mathrm{~g}$ of Amoxicillin trihydrate and $0.01 \mathrm{~g}$ of Clavulanate Lithium were weighed and transferred into a $50 \mathrm{ml}$ volumetric flask. Distilled water was then added up to the $50 \mathrm{ml}$ mark to produce a final concentration of $0.05 \% \mathrm{w} / \mathrm{v}$ or $0.5 \mathrm{mg} / \mathrm{ml}$ of amoxicillin trihydrate and $0.02 \% \mathrm{w} / \mathrm{v}$ or $0.2 \mathrm{mg} / \mathrm{ml}$ of clavulanate lithium. $20 \mu \mathrm{l}$ volumes of both standards and samples were injected into High Performance Liquid Chromatography (HPLC). An Agilent 1100 system was used and conditions for all HPLC analysis were as follows: Mobile Phase-5 volumes of methanol, 95 volumes of $0.78 \% \mathrm{w} / \mathrm{v}$ Sodium dihydrogen phosphate monohydride ( $\mathrm{pH} 4.4$ ); Flow rate of $2 \mathrm{ml} / \mathrm{min}$ using isocratic elution; $150 \times 4.6 \mathrm{~mm}$ C18 Supelco Column. Detection wavelength $-220 \mathrm{~nm}$. Calculations were used to convert the amount of Potassium Clavulanate to Clavulanic acid and Amoxicillin Trihydrate to amoxicillin.

\subsection{Content Analysis}

The content of amoxicillin and clavulanic acid in each formulation was determined using HPLC. For each tablet pack three tablets were separately weighed and each placed in a $100 \mathrm{ml}$ volumetric flask. $50 \mathrm{ml}$ of distilled water was measured into each of the flasks, which was then placed in a mechanical shaker for ten minutes to ensure that the tablet had completely disintegrated. Each of the flasks was thereafter made to volume with distilled water and placed in a Clifton ultrasonic bath for 3 minutes to obtain a uniformly mixed solution. $5 \mathrm{ml}$ of the solution was then withdrawn, transferred into a $50 \mathrm{ml}$ volumetric flask and made to volume with distilled water to give an expected final concentration of $0.05 \% \mathrm{w} / \mathrm{v}$ of amoxicillin trihydrate and $0.02 \% \mathrm{w} / \mathrm{v}$ of potassium clavulanate. The $50 \mathrm{ml}$ volumetric flask was manually shaken for a few minutes and some drug solution drawn and filtered into an HPLC vial using a $0.45 \mu \mathrm{m}$ filter. Two injections were made per sample.

The procedure for the injection formulations was similar to that for the tablets, however because the injections are already in powder form, they cannot be weighed directly because of the risk of losing some powder. Therefore each vial containing drug powder was weighed. The vial was then filled with distilled water and the contents transferred to a $100 \mathrm{ml}$ volumetric flask. The vial was washed three times with distilled water and transferred to the volumetric flask. The flask was finally made to volume with distilled water. The empty vial was dried, weighed, and the weight of the weight of the drug obtained by difference.

The volumetric flask was then placed in a Clifton ultrasonic bath for 3 minutes for uniform mixing. Similarly, $5 \mathrm{ml}$ of the resulting solution was drawn using a pipette, placed in a $50 \mathrm{ml}$ volumetric flask, and topped up to the $50 \mathrm{ml}$ mark with distilled water to obtain a final concentration of $0.05 \% \mathrm{w} / \mathrm{v}$ of amoxicillin trihydrate and $0.02 \%$ w/v of potassium clavulanate. The $50 \mathrm{ml}$ volumetric flask was manually shaken for a few minutes and some drug solution drawn and filtered into an HPLC vial. Two injections were made per sample.

For analysis of suspension formulations, the samples were analyzed based on British Pharmacopoeia (BP) requirements. Twenty-two (22) samples from five (5) brands were analyzed in all. Reconstitution of the powder for suspension was made according to the manufacturer's specifications. The suspensions were further diluted with distilled water to a final concentration of $0.05 \% \mathrm{w} / \mathrm{v}$ of amoxicillin trihydrate and $0.02 \% \mathrm{w} / \mathrm{v}$ of potassium clavulanate. The amounts of clavulanic acid and amoxicillin were calculated from potassium clavulanate and amoxicillin trihydrate respectively.

\subsection{Dissolution Test}

Two dissolution station apparatus: ERWEKA DT-600 and DT-800 were calibrated in the paddle mode and set 
to a temperature of $37^{\circ} \mathrm{C}+/-0.5^{\circ} \mathrm{C}$, time duration of 30 minutes and a frequency of 75 rotations per minute (rpm). A set volume of $900 \mathrm{ml}$ of dissolution medium, distilled water, was accurately measured using a $1000 \mathrm{ml}$ measuring cylinder and poured into each of the six glass vessels and maintained at a temperature of $37^{\circ} \mathrm{C}+/-0.5^{\circ} \mathrm{C}$. Standard thermometers were placed in each vessel to crosscheck the temperature. Three tablets of brand A, from one location were placed into three vessels and another three tablets of the same brand from a different area were placed into the other three vessels. This was repeated for all the brands available, hence, six tablets of the same brand were placed into the buffer/dissolution medium (distilled water) all at the same time. At the end of the run time, $10 \mathrm{ml}$ was sampled from each vessel into a labeled beaker with a calibrated syringe for further analysis. The drug solutions were allowed to equilibrate to room temperature and portions filtered and transferred into agilent HPLC vials. Two injections were made per sample.

\subsection{Antimicrobial Effect of Amoxicillin/Clavulanic Acid Formulations}

Bacillus subtilis and Klebsiellaspp were uncoded clinical isolates obtained from the Korle-bu Teaching Hospital in Accra, Ghana. Salmonella typhi (ATCC 33458), Escherichia coli (NCTC 1351) and Staphylococcus aureus (NCTC 6571) were gifts from the Noguchi Memorial Institute, Legon, Ghana. Stock suspensions of the antibiotics were prepared and used to make concentrations of $0.001 \%, 0.002 \%, 0.004 \%$ and $0.008 \% \mathrm{w} / \mathrm{v}$ in sterile water. Nutrient agar was prepared and $25 \mathrm{ml}$ portions were transferred into test tubes. The test tubes were then sterilized in an autoclave for 15 minutes at $121^{\circ} \mathrm{C}$ and stabilized in a water bath at $45^{\circ} \mathrm{C}$ to prevent the molten nutrient agar from solidifying.

Stabilized agar in test tubes was seeded with $1 \mathrm{ml}$ each of a 24 hour broth cultured bacteria $\left(1.2 \times 10^{6} \mathrm{cfu} / \mathrm{ml}\right)$. The bacteria used were B. subtilis, Klebsiella sp, Salmonella sp, E. coli, and S. aureus. Seeded molten agar was aseptically transferred into petri dishes and allowed to set at room temperature. Using the $10 \mathrm{~mm}$ diameter cork borer, 4 holes were made in the agar with reasonable spacing.

Three of the holes were each filled with the $0.1 \mathrm{ml}$ of antibiotic suspension and the fourth with sterile water. The plates were covered and left on the bench for an hour after which they were incubated in an upright position at $37^{\circ} \mathrm{C}$ for 24 hours. Zones of inhibition (ZOI) were measured as the diameter of clear area of no growth around the edges of the wells. The above procedure was repeated for the next three concentrations of the same sample and for the other samples. Plots of drug concentration versus
ZOI were made and the minimum inhibitory concentration (MIC) was obtained as the intercept on the concentration axis, of the line of best fit. The MIC values obtained, though directly correlate with the susceptibility of an organism to the drug, are interpreted here to also correspond to the concentration on the drug in solution. The identity of the drug molecule and the concentration in the various formulations has been established by in vitro content and dissolution tests. Sample MIC values were compared with published MIC values for the various organisms [12]. A significantly lower sample MIC value is an indication of low drug concentration.

\subsection{Statistical Analysis}

The statistical tool used was the independent samples T-test.

\section{Results}

\subsection{Disintegration Test}

The results of the disintegration test are presented in Figure 1. All the samples passed the B.P specification for disintegration test [13]. The highest disintegration time recorded was 14.11 minutes for DT-E with the least being 6.43 minutes for ET-C. All the other samples had variable disintegration times but all fell within the specified time range. From the results obtained it can be inferred that all the tablets will be able to readily release the active ingredients in aqueous medium or in gastrointestinal fluid.

\subsection{Content Assay and Dissolution Test}

In order to verify if the HPLC equipment was good enough for carrying out experiments, the system suitability of the equipment was determined by calculating the relative standard deviation. Relative standard deviations below 2\% are acceptable [13] for the dissolution and content assay of the tablets and injectables. The relative standard deviation for both amoxicillin and clavulanic acid was below $2 \%$ in either the dissolution or content system suitability test.

According to the BP, the acceptable content range of a newly reconstituted suspension should be between $80 \%$ and $120 \%$ of the label claim when stored at the appropriate temperature and period stated for use. A sample has to pass for both amoxicillin and clavulanic acid in order to be considered as having passed content analysis. Content analysis was performed for all the five (5) brands of amoxicillin/clavulanic acid suspension obtained. Only one (1) out of seven (7) samples (14.3\%) of Brands A or $B$ passed assay (Figures 2 and 3). The two samples that passed were purchased from Agona Swedru and Cape Coast respectively. Four (4) out of five (5) samples (80\%) 


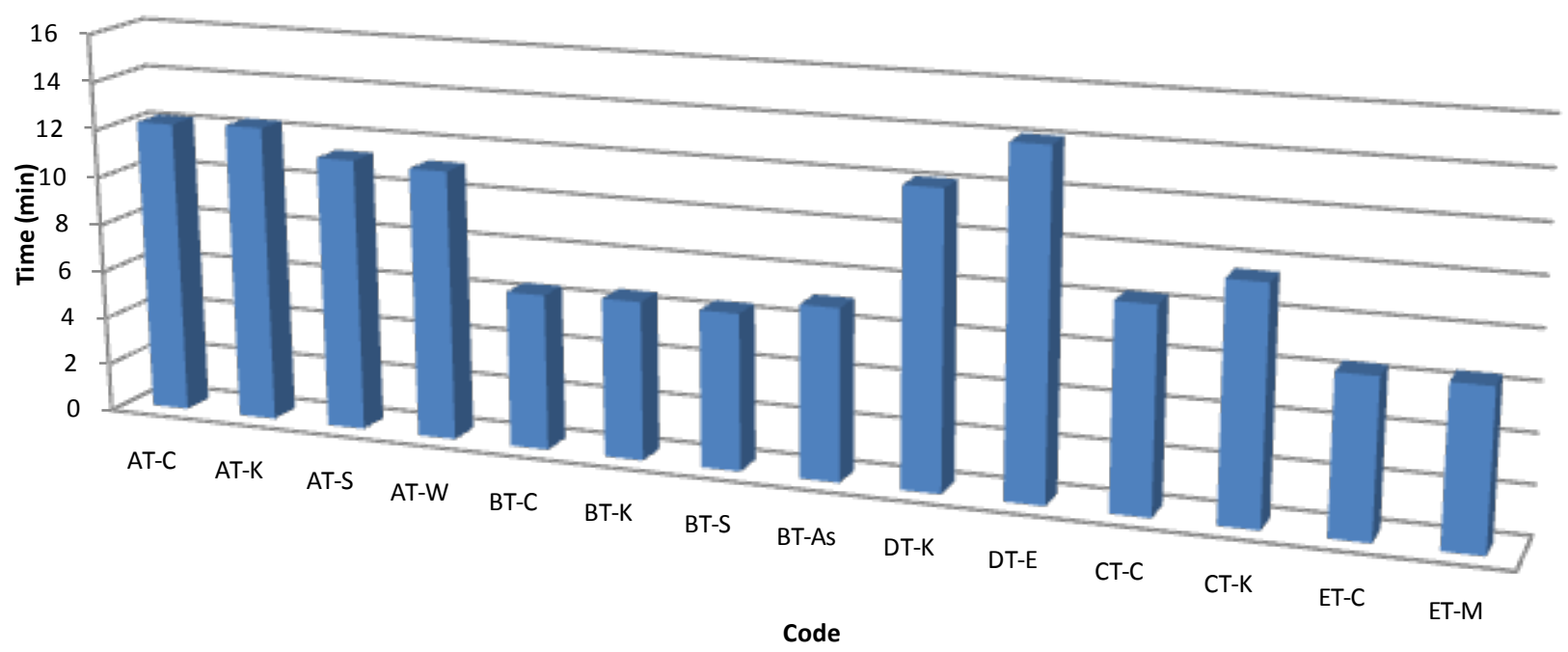

Mean Disintegration time (min)

Figure 1. Disintegration time of samples.

Brand A

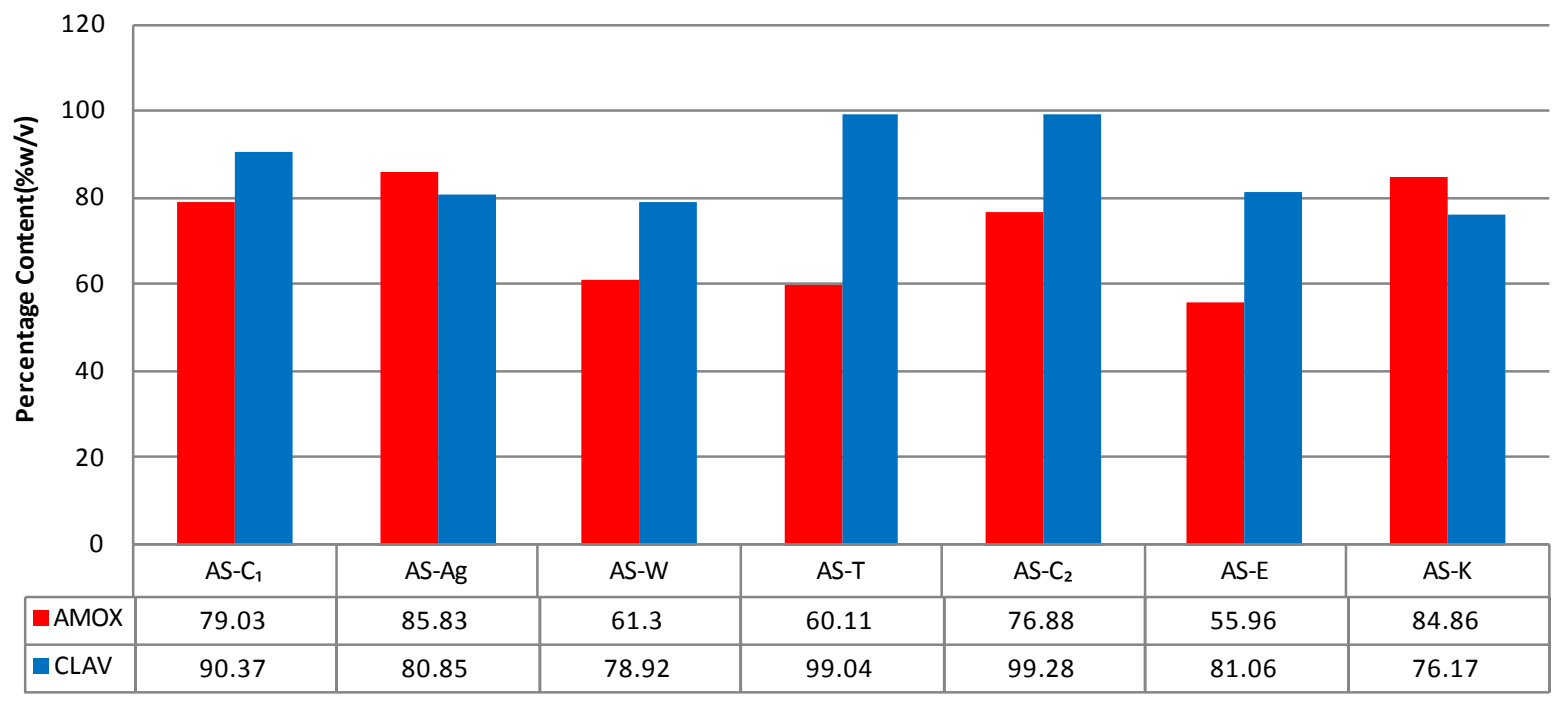

Figure 2. Percentage of amoxicillin/clavulanic acid content of brand A suspensions.

of Brand C passed (Figure 4). All (100\%) of brands D and E passed (Figures 5 and 6). A summary of the results from the analysis of the suspension dosage forms showed that only nine (9) of the twenty-two (22) samples passed contentanalysis (41\%). A statistically significance difference was observed when the content of clavulanic acid was compared in all suspension samples ( $<<0.01)$; however differences in amoxicillin content was not $(\mathrm{p}>$ 0.01) (Table 1).

Content assay was doneon all five (5) brands of amoxicillin/clavulanic acid tablets obtained from fourteen (14) different pharmacies in seven (7) districts as well as two
(2) brands of injectables obtained from four (4) pharmacies in three (3) districts. The acceptance criteria for the assay of amoxicillin/clavulanic tablets and injectables are between $90 \%$ and $120 \%$ and each tablet should pass for both ingredients [13]. All the tablets failed content analysis for amoxicillin and twelve (12) out of fourteen (14) tablet batches (86\%) passed for clavulanic acid alone (Figure 7). A statistically significance difference was observed when the content of amoxicillin was compared in all tablet samples ( $p<0.01$ ); however differences in clavulanic acid contentwas not ( $p>0.01$ ) (Table 1$)$. Analysis of the injectables showed all samples passed for 


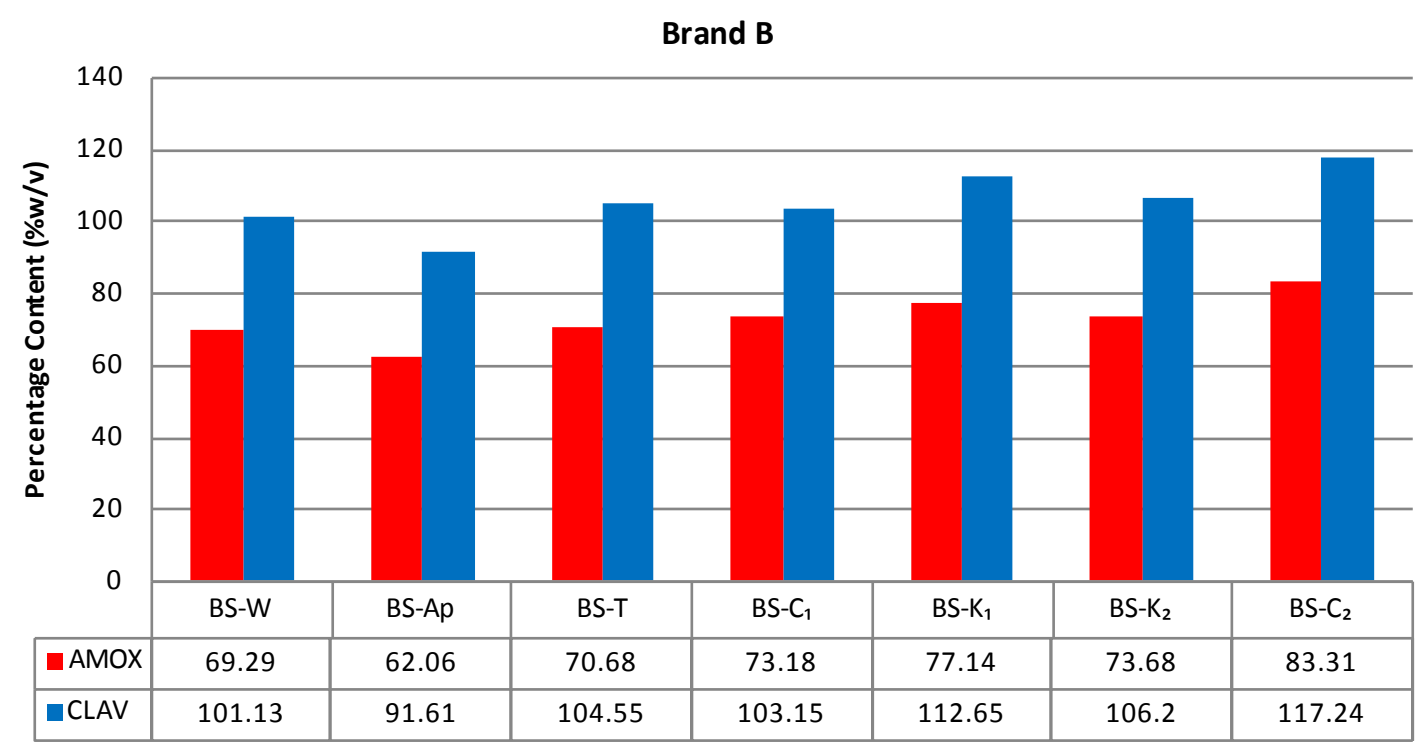

Figure 3. Percentage of amoxicillin/clavulanic acid content of brand B suspensions.

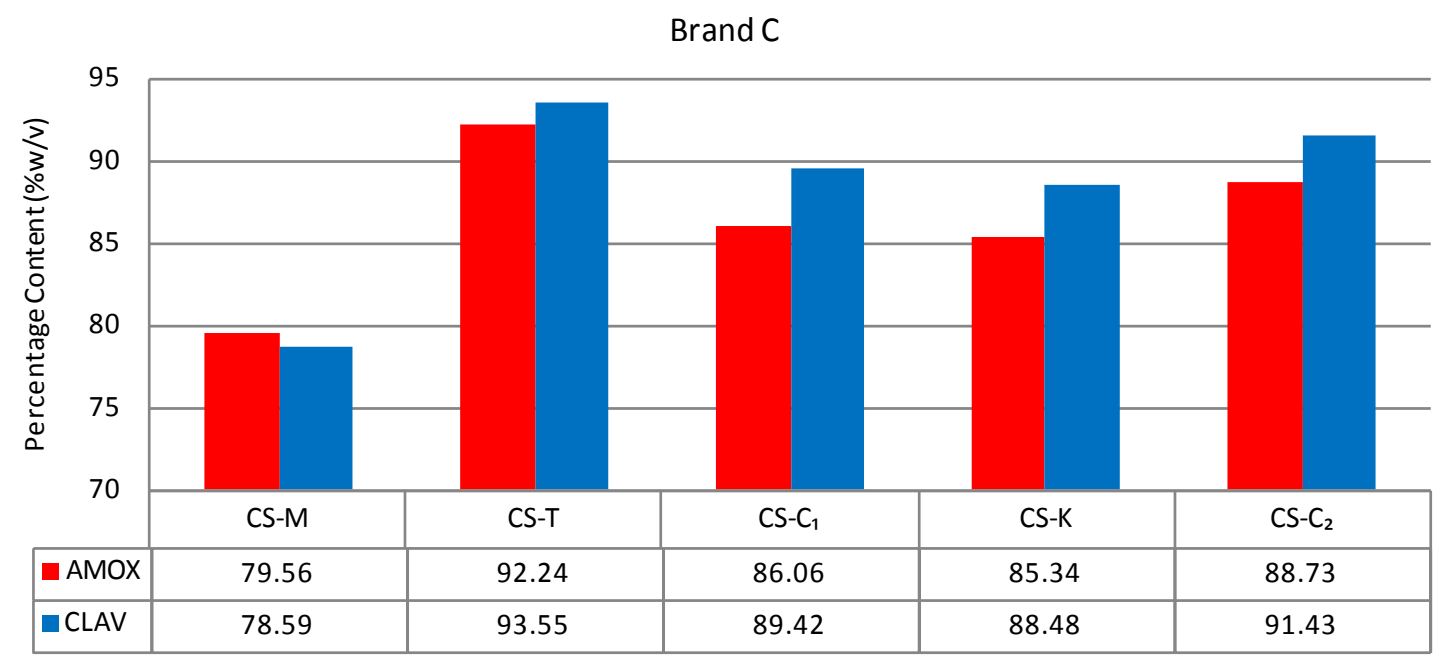

Figure 4. Percentage of amoxicillin/clavulanic acid content of brand C suspensions.

Brand D

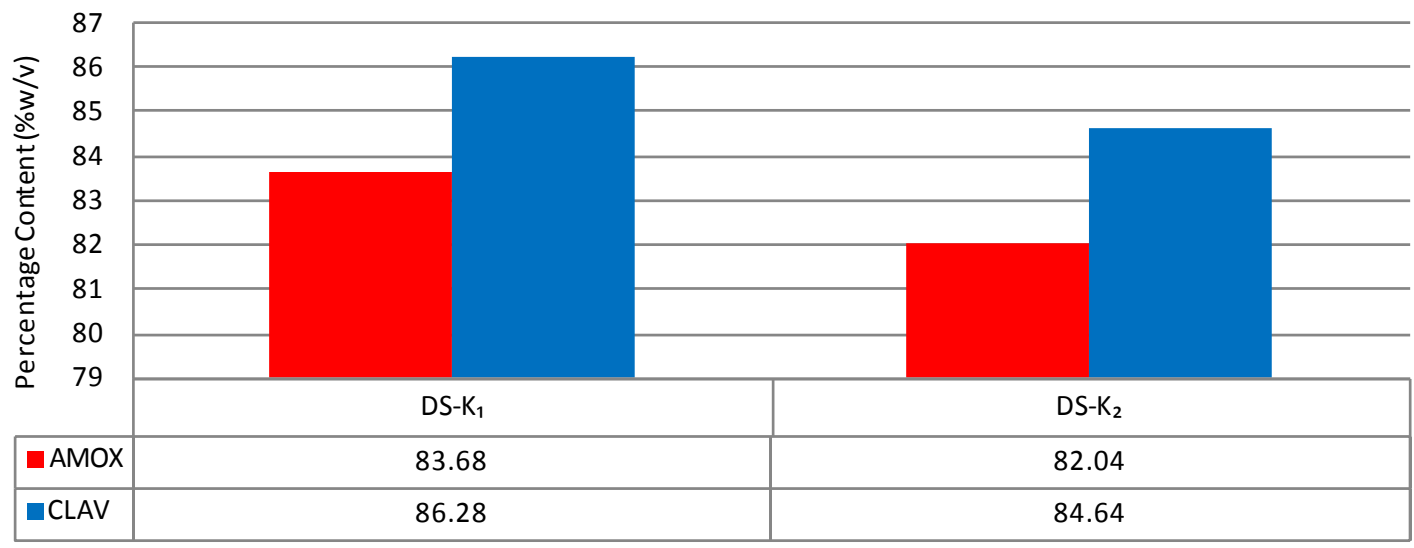

Figure 5. Percentage of amoxicillin/clavulanic acid content of brand D suspensions. 


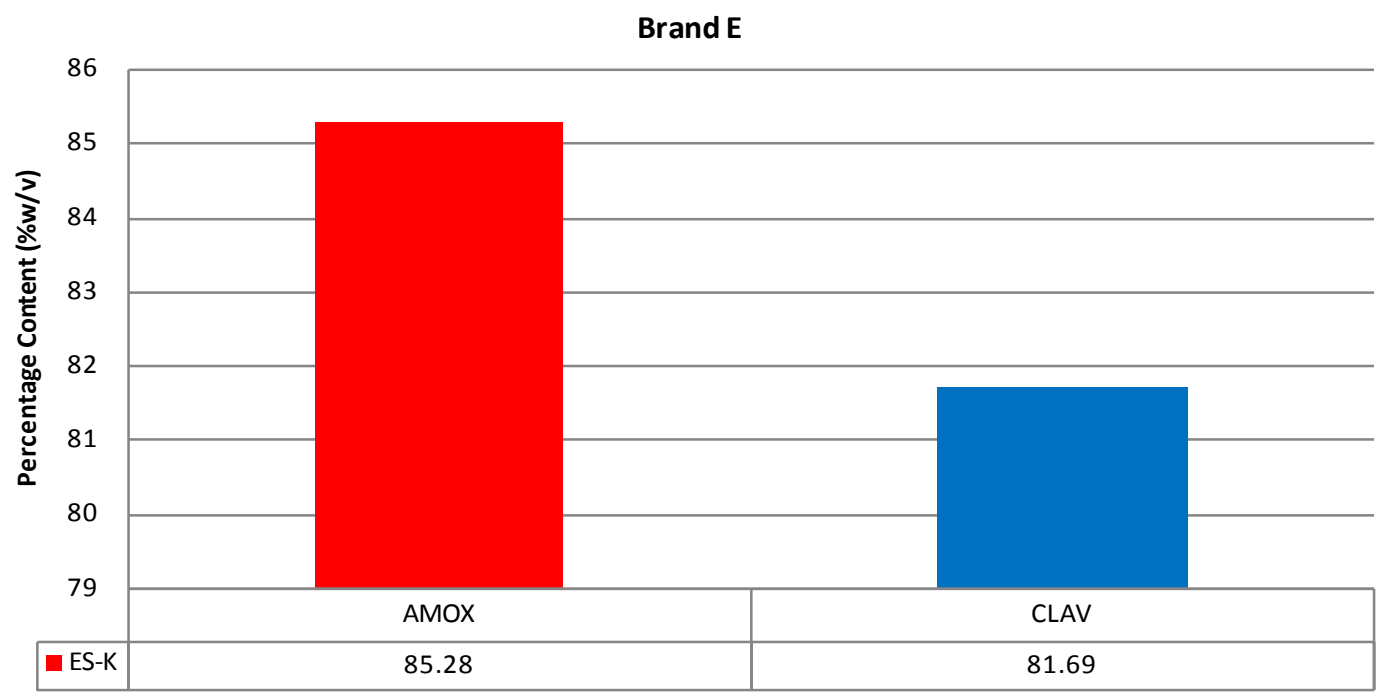

Figure 6. Percentage of amoxicillin/clavulanic acid contentof brand E suspension.

Table 1. One-sample test of amoxicillin/clavulanic acid formulations. Significance $=\mathbf{0 . 0 1}$. Test values are the lower limits of reference concentration ranges.

\begin{tabular}{|c|c|c|c|c|c|c|c|}
\hline & & $\mathbf{N}$ & Mean & Std. deviation & $\mathbf{t}$ & df & $\mathbf{p}$ \\
\hline & & & & lue $=80$ & & & \\
\hline \multirow[t]{3}{*}{ Suspension } & Amoxicillin & 22 & 77.1018 & 10.17178 & -1.336 & 21 & 0.196 \\
\hline & Clavulanic acid & 22 & 92.5591 & 11.52484 & 5.111 & 21 & 0.000 \\
\hline & \multicolumn{7}{|c|}{ Test value $=90$} \\
\hline \multirow[t]{3}{*}{ Tablet Content } & Amoxicillin & 14 & 75.585 & 5.71205 & -9.442 & 13 & 0.000 \\
\hline & Clavulanic acid & 14 & 91.54 & 18.09205 & 0.318 & 13 & 0.755 \\
\hline & \multicolumn{7}{|c|}{ Test value $=75$} \\
\hline \multirow[t]{2}{*}{ Tablet Dissolution } & Amoxicillin & 14 & 79.8886 & 21.1786 & 0.864 & 13 & 0.403 \\
\hline & Clavulanic acid & 14 & 84.5981 & 23.56951 & 1.524 & 13 & 0.152 \\
\hline
\end{tabular}

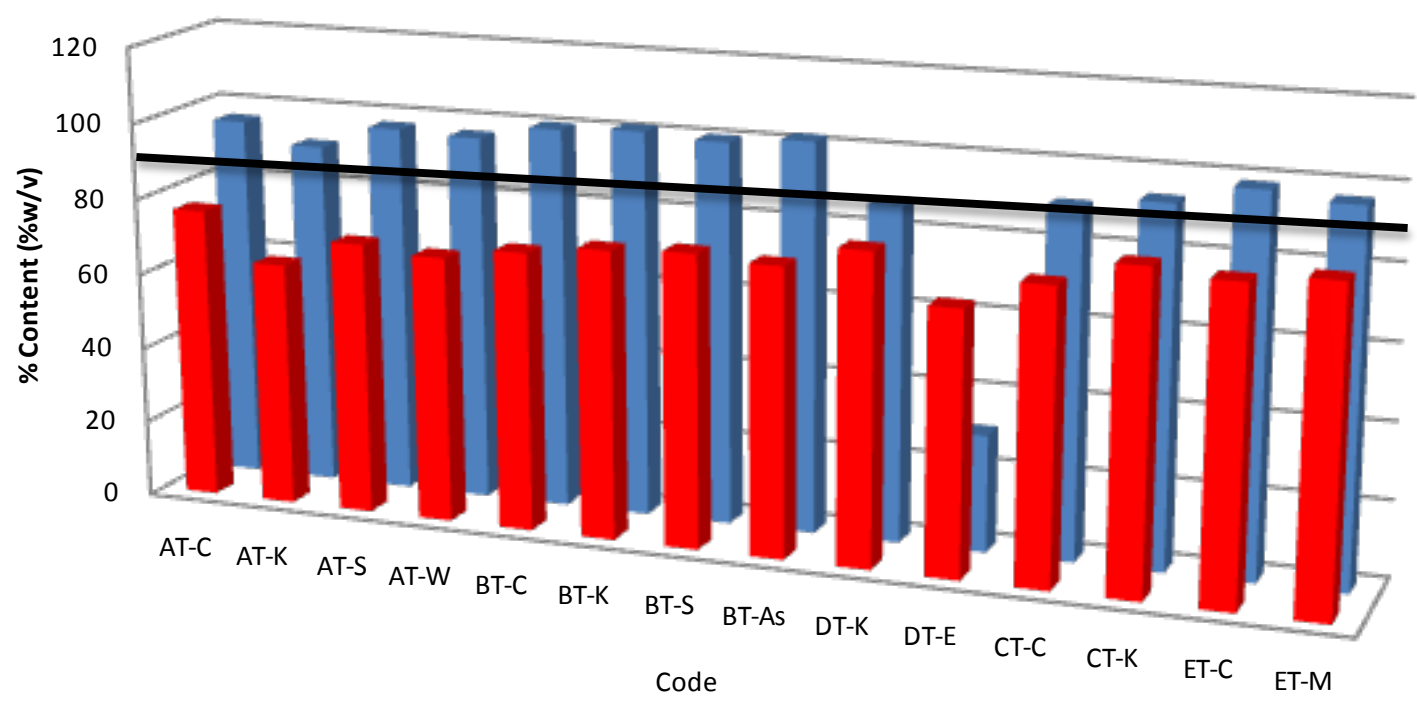

Figure 7. Percentage of amoxicillin/clavulanic acid content in tablet samples after content assay. The solid horizontal line represents the lower acceptable limit for drug content per BP. 
clavulanic acid but failed for amoxicillin content (Figure 8). Since no tablet or injectable passed both amoxicillin and clavulanic acid content per B.P criteria, there was $100 \%$ failure. A study by Sengaloundeth et al. in 2009 found a number of fake and substandard oral artesunate dosage forms in Laos [14]. Later on in 2011, some of the authors in the Laos drug quality study thought it necessary to expand the scope of their investigations. They determined in their search that most of the counterfeit and substandard anti-malarials found in Africa have their origins in South East Asia. In that study, they found numerous fake and substandard anti-malarials in most of the African countries they sampled from, including Ghana, They however, stated a major limitation in their study as the lack of dissolution studies [15]. This prompted the researchers in the present study to include tablet dissolution studies as well as in vitro efficacy studied since we were dealing with antibiotics. Prior to this study, a pilot study was done by one of the researchers in Accra, a major city in Ghana. In that study, some substandard Artemether-Lumefantrine tablets were found in that region [16].

Dissolution test was done on all the tablets used for drug content analysis. The B.P 2013 stipulates that at 30 minutes, all tablets should have released into the dissolution medium an amount not less than $75 \%$ of the labeled amount of either amoxicillin and clavulanic acid. Thirteen (13) of all tablet batches tested (93\%) passed the test (Figure 9). One batch (DT-E) failed for both amoxicillin and clavulanic acid, showing only an average of 3.36\% and $7.30 \%$ respectively. Statistical analysis of all tablet samples for dissolution showed that the difference among them was not significant ( $p>0.01$ ) (Table 1).

\subsection{Anti-Microbial Effect of Amoxicillin/Clavulanic Acid Samples}

A subset of the tablet, injectable and suspension formulations of amoxicillin/clavulanic samples obtained were used for in vitro anti-microbial efficacy studies. Not all the samples obtained were tested because most had been used up in previous tests. A low MIC is highly significant and indicative of a potent product which has an effective therapeutic outcome. The result of the antimicrobial susceptibility test showed that all samples tested had significant inhibitory effect against $B$. subtilis except for samples AS- $\mathrm{C}_{1}$ and DT-E which showed very minimal activity (Table 2). The means of all samples, when

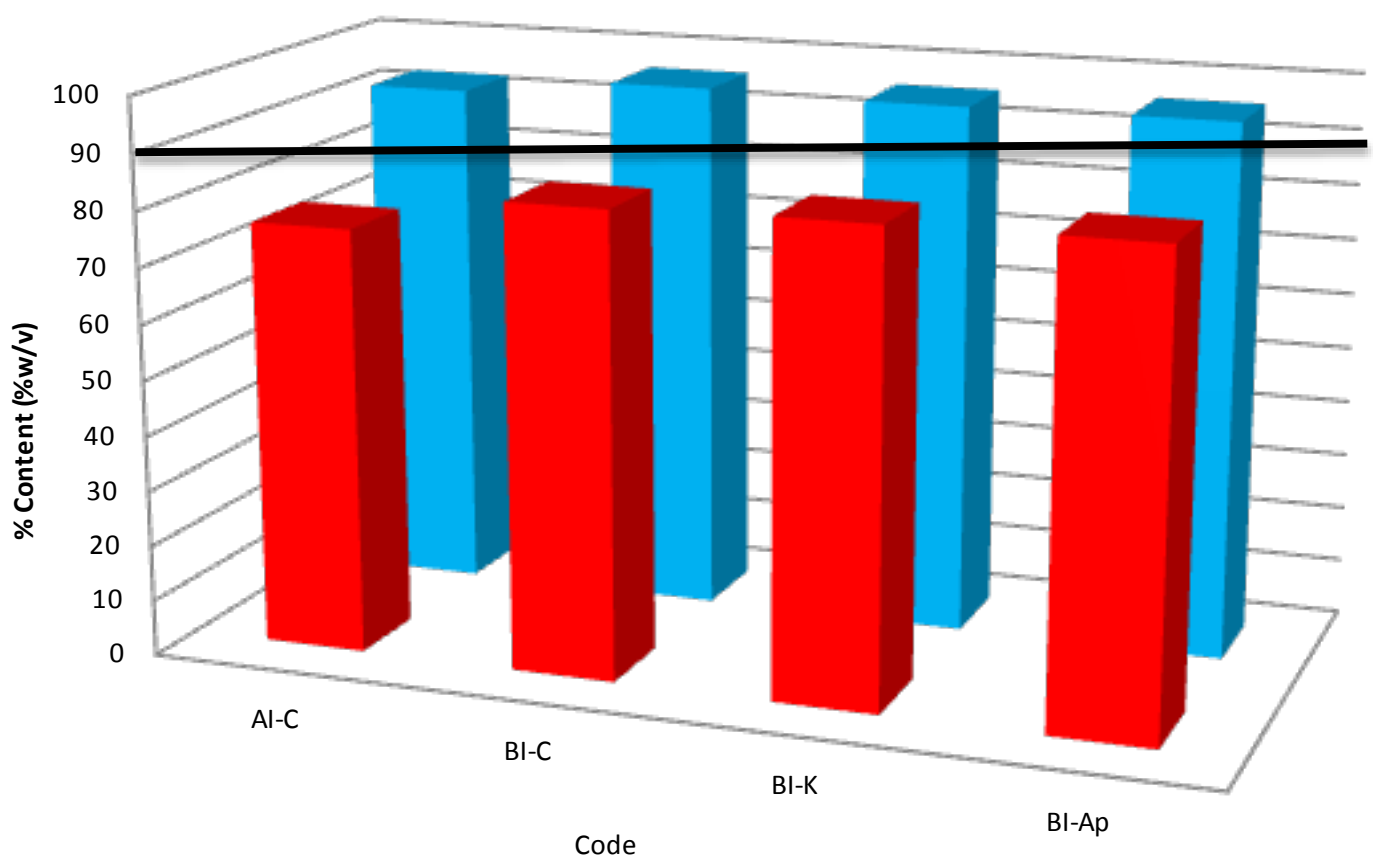

\begin{tabular}{|l|c|c|c|c|}
\cline { 2 - 5 } \multicolumn{1}{c|}{} & AI-C & BI-C & BI-K & BI-Ap \\
\hline \#Amoxycillin & 76.45 & 83.35 & 84.2 & 84.56 \\
\hline EClavulanic & 92.95 & 96.08 & 95.62 & 95.9 \\
\hline
\end{tabular}

Figure 8. Percentage of amoxicillin/clavulanic acid content in injectable samples after content assay. The solid horizontal line represents the lower acceptable limit for drug content per BP. 


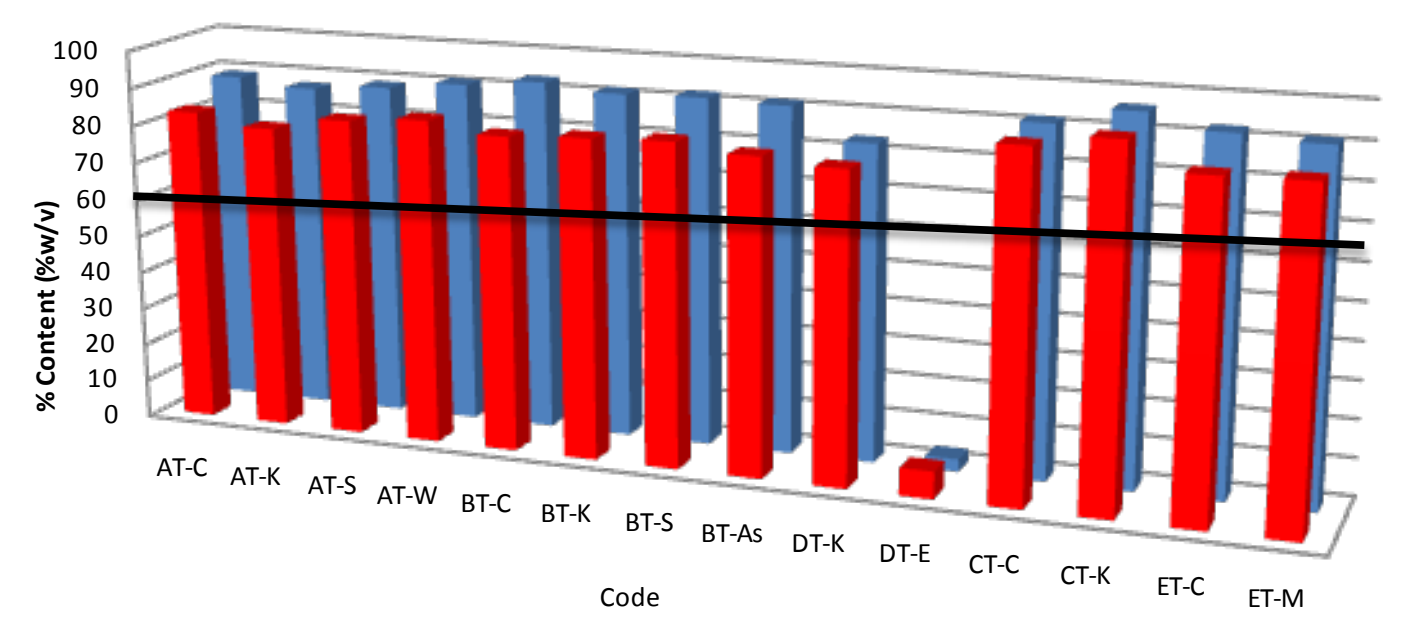

\begin{tabular}{|l|c|c|c|c|c|c|c|c|c|c|c|c|c|c|}
\hline & AT-C & AT-K & AT-S & AT-W & BT-C & BT-K & BT-S & BT-As & DT-K & DT-E & CT-C & CT-K & ET-C & ET-M \\
\hline -Amoxycillin & 83.49 & 80.61 & 84.29 & 85.93 & 83.35 & 84.44 & 85.26 & 83.25 & 81.78 & 7.3 & 90.72 & 93.86 & 86.86 & 87.3 \\
\hline -Clavulanic Acid & 89.073 & 87.3 & 89.02 & 91.25 & 93.31 & 91.7 & 92.2 & 91.63 & 83.57 & 3.36 & 91.81 & 96.23 & 92.55 & 91.37 \\
\hline
\end{tabular}

Figure 9. Percentage of amoxicillin/clavulanic acid content in tablet samples after dissolution testing. The solid horizontal line represents the lower acceptable limit for drug content per BP.

compared to the reference concentration of $4 \mu \mathrm{g} / \mathrm{ml}$ showed differences that were statistically significant $(\mathrm{p}<$ 0.05 ) (Table 3). All samples tested showed inhibitory effect against Klebsiella sp, Salmonella spand E. coliexcept DT-E which was least effective (Table 2). The mean differences between the samples and reference were statistically significant $(\mathrm{p}<0.05)$ (Table 3). Staphylococcus aureus, with a reference concentration of $4 \mu \mathrm{g} / \mathrm{ml}$ for susceptibility, showed the most resistance to the samples tested (Table 2). The mean differences between the samples and reference were not statistically significant $(\mathrm{p}>$ 0.05 ) (Table 3). Also analysis revealed relatively higher MIC values for sample $a x$-std, which was amoxicillin powder and used as a standard. This is because sample $a x$-std, had no clavulanic acid and was subject to degradation by $\beta$-lactamase produce by gram positive bacteria.

\section{Discussions}

Tablet disintegration is the first step for a drug to become bioavailable. A tablet must first disintegrate and release the drug molecule to the gastrointestinal fluids. The active ingredient in the disintegrated tablet must dissolve in gastrointestinal fluid to be absorbed into the blood stream. Tablets should thus be sufficiently hard to withstand handling without crumbling or breaking but they should also be sufficiently soft for easy disintegration in the stomach or intestine in order to make the drug available to the body. Tablets may be too hard and fail the disintegration test because of poor manufacturing process or incorrect storage. The disintegration test thus measures the time taken for tablets to disintegrate into particles. This is a necessary condition for drug dissolution and could be the rate determining step in the process of drug absorption. Tablet disintegration is thus a factor of consideration in the bioavailability of drug because it is directly proportionalto the rate of dissolution of the dosage form. The BP stipulates a disintegration time of not more than 15 minutes for uncoated tablets [13]. All the tablet formulations passed the disintegration test and as such, it will be expected that the active ingredient will be released in time for dissolution to start in the stomach.

Assay of content of both tablet and injectable formulations produced rather disappointing results. All the samples tested failed to meet B.P specifications for drug assay. The samples failed, not because they did not have the active ingredients, but rather the assayed amounts fell short of the label claim. All the samples had at least $60 \%$ of either amoxicillin or clavulanic acid except for sample DT-E which had clavulanic acid content of $30 \%$. A host of factors may affect the content of a drug product. Factors that may affect the quality of manufactured drug products include storage temperature conditions in which drug products are stored, level of moisture at these storage sites (humidity), exposure to light, microbial contamination, packaging materials used for the drug product, transportation factors associated with the distribution of the drug product, components of drug composition and most importantly, the nature of the active ingredient used 
Table 2. In vitro efficacy of amoxicillin/clavulanic acid tablets, Suspensions and injectables. Interpretation of MIC values for Klebsiella sp, Salmonella sp, and E. coli are: $\leq 4-8 \mu \mathrm{g} / \mathrm{ml}=$ Susceptible (S); $9-16 \mu \mathrm{g} / \mathrm{ml}=$ intermediate (I); $\geq 17-32 \mu \mathrm{g} / \mathrm{ml}=$ Resistant (R). MIC values for B. subtilis and $S$. aureus are: $\leq 2-4 \mu \mathrm{g} / \mathrm{ml}=$ Susceptible (S); $\geq 5-8 \mu \mathrm{g} / \mathrm{ml}=\mathrm{Resistant}(\mathrm{R})$.

\begin{tabular}{|c|c|c|c|c|c|}
\hline \multirow{2}{*}{ Sample code } & \multicolumn{5}{|c|}{ Organism name/sample concentration ( $\mu \mathrm{g} / \mathrm{ml})$} \\
\hline & B. subtilis & Klebsiellasp & Salmonella sp & E.coli & Staph. aureus \\
\hline Ax std & 4.11 & & & 0.00292 & 18.6 \\
\hline AT-K & 2.78 & 0.98 & 2.98 & 2.61 & 2.85 \\
\hline AS- $C_{1}$ & 8.4 & 2.35 & 3.76 & 0.292 & 9.19 \\
\hline AS- $C_{2}$ & 0.0186 & 0.00104 & 0.0607 & 1.90 & 0.191 \\
\hline AS-Ag & 0.93 & 0.53 & 2.15 & 0.91 & 1.39 \\
\hline AS-T & 1.48 & 1.72 & 0.0057 & 0.43 & 3.01 \\
\hline AS-W & 1.34 & 5.60 & 1.74 & 2.98 & 4.46 \\
\hline BT-C & 1.05 & 1.48 & 0.048 & 2.64 & $9.22 * 10^{-10}$ \\
\hline BT-As & 2.94 & 0.86 & 0.78 & 0.29 & 3.17 \\
\hline BT-S & 2.73 & 0.065 & 1.73 & 4.05 & 1.31 \\
\hline BS-C & 1.12 & 3.36 & 1.70 & 5.48 & 5.26 \\
\hline BS-K $K_{1}$ & 0.28 & 0.047 & 2.86 & 1.66 & 2.60 \\
\hline BS- $\mathrm{K}_{2}$ & 3.30 & 2.28 & 0.23 & 3.64 & 1.76 \\
\hline BS-W & 2.6 & 1.65 & 0.00369 & 0.00375 & 3.01 \\
\hline BI-C & 0.38 & 1.66 & 2.91 & 0.61 & 0.039 \\
\hline BI-K & 1.33 & 0.52 & 1.20 & 4.05 & 1.31 \\
\hline CT-C & 2.24 & 2.26 & 0.000497 & 1.51 & 0.716 \\
\hline CT-K & 3.09 & 1.70 & 2.18 & 1.13 & 3.90 \\
\hline CS-C ${ }_{1}$ & 1.01 & 4.60 & 3.34 & 2.69 & 8.86 \\
\hline $\mathrm{CS}-\mathrm{C}_{2}$ & 1.99 & 0.15 & 1.26 & 1.38 & 5.72 \\
\hline CS-M & 1.05 & 2.45 & 1.43 & 2.65 & 8.11 \\
\hline CS-T & 1.99 & 0.15 & 1.26 & 1.24 & 2.80 \\
\hline DT-C & 1.9 & 2.01 & 0.625 & 1.86 & 0.00363 \\
\hline DT-E & 13.45 & 14.12 & 14.12 & 6.14 & 14.51 \\
\hline DT-K & 3.21 & 1.82 & 0.77 & 3.06 & 3.14 \\
\hline DS-K ${ }_{1}$ & 2.37 & 1.63 & 2.51 & 4.76 & 7.81 \\
\hline DS-K ${ }_{2}$ & 0.87 & 0.82 & 2.19 & 2.13 & 0.29 \\
\hline ET-C & 1.18 & 1.81 & 1.99 & 2.83 & 4.01 \\
\hline ET-M & 0.29 & 0.00032 & 1.39 & 0.699 & 0.399 \\
\hline ES-K & 2.21 & 2.07 & 0.024 & 2.41 & 1.72 \\
\hline ES-K 1 & 1.89 & 0.58 & 1.30 & 2.24 & 3.98 \\
\hline $\mathrm{ES}-\mathrm{K}_{2}$ & 3.10 & 1.47 & 1.70 & 1.48 & 3.14 \\
\hline
\end{tabular}

Table 3. One-sample test of antimicrobial susceptibility to antibiotics. Test values are the lower limits of reference susceptibility concentrations.

\begin{tabular}{|c|c|c|c|c|c|c|}
\hline & $\mathbf{N}$ & Mean & Std. deviation & t & df & Sig. (1-tailed) \\
\hline \multicolumn{7}{|c|}{ Test value $=4$} \\
\hline B. subtilis & 32 & 2.3946 & 2.53981 & -3.576 & 31 & 0.001 \\
\hline S. aureus & 32 & 3.9768 & 4.21354 & -0.031 & 31 & 0.975 \\
\hline \multicolumn{7}{|c|}{ Test value $=8$} \\
\hline Klebsiella sp & 31 & 1.9595 & 2.5984 & -12.943 & 30 & 0.000 \\
\hline Salmonella sp & 31 & 1.879 & 2.50943 & -13.581 & 30 & 0.000 \\
\hline E. coli & 32 & 2.17993 & 1.55635 & -21.154 & 31 & 0.000 \\
\hline
\end{tabular}


for the formulation. All these factors eventually affect the drug product's stability. Drug Stability refers to the capacity of a drug substance or product to remain within established specifications of identity, strength, quality, and purity in a specified period of time [17]. The most plausible explanation for the failure of the samples is the poor storage conditions that these drugs might have been subjected to. Most of the drugs are kept in trucks and vans under the scorching sun and without refrigeration for days. Content analysis of the suspension dosage forms showed only $41 \%$ passed and this could also be attributed to poor storage and/or manufacturing conditions.

Dissolution of a drug is prerequisite for absorption of the drug into the body and the dissolution rate is directly related to the bioavailability. For this reason, dissolution testing is commonly used as a tool to compare the bioavailability of the same drugs manufactured by different companies. Dissolution is the primary quality control test to determine whether a drug product can release its active pharmaceutical ingredient(s) in a timely manner [18]. It is thus required for all solid oral dosage forms in which absorption of the drug is necessary for the product to exert the desired therapeutic effect. If the rate of dissolution is the rate-limiting step, then the dissolution rate determines the bioavailability. Dissolution is thus used as a quality control measure for batch release, to ensure continued quality during the shelf life and also to detect batches with poor quality without rejecting batches of adequate quality [19]. All the brands studied passed for dissolution with the exception of sample DT-E. Sample DT-E contained an average of 3.36\% w/v of amoxicillin and $7.30 \% \mathrm{w} / \mathrm{v}$ of clavulanic acid. This may be attributed to factors that may have influenced the dissolution of the active pharmaceutical ingredient (API) from the dosage form which may include the nature of the excipients used in compounding or the formulation process. Although sample DT-E passed disintegration test, it registered the longest time, and this could have influenced the rate of dissolution. DT-E also produced the lowest API content among all samples analyzed which definitely affects the results of the dissolution test. It is worthwhile to note that although all the tablet dosage forms analyzed failed content analyses, 93\% passed dissolution assay. A possible explanation for this observation is the less stringent lower limit (75\%) for a tablet to pass the dissolution test. One can safely infer from the results that the drug products that passed the dissolution test may produce therapeutic levels of API. This will not always be the case if API degradation, due to poor storage conditions, is the cause of the failed drug content. With time, the API levels will reduce to such a level that dissolution tests will fail. Since dissolution tests are not done for suspensions, we can safely conclude that those that failed content analysis will have reduced bioavailability. In vitro efficacy tests confirmed results obtained for content analysis and dissolution tests. Samples that generally showed lower content showed higher MIC values, hence will be less efficacious.

\section{Conclusion}

All the samples analyzed for drug content failed to meet compendia requirements and are therefore considered of poor quality. This conclusion can be drawn even though some of them passed disintegration, dissolution or in vitro microbial growth inhibitory tests. Most of the brands had more than sixty percent of either amoxicillin or clavulanic acid content and would likely be substandard rather than counterfeit drugs. It is therefore necessary to enforce strict regulatory laws that will control the quality of drugs from the time they are manufactured until they get to the consumer. Stability tests required for the zone in which the drug would be marketed should be ensured. Random sampling of drugs on the market should also be stepped up.

\section{Acknowledgements}

We wish to express our sincere thanks to the directors and technicians at the Ghana Food and Drugs Authority for their immense assistance in our HPLC analysis and Dissolution tests.

\section{REFERENCES}

[1] M. E. Arroliga and L. Pien, "Penicillin Allergy: Consider Trying Penicillin Again,” Cleveland Clinic Journal of Medicine, Vol. 70, No. 4, 2003, pp. 313-314. http://dx.doi.org/10.3949/ccjm.70.4.313

[2] C. Walsh, "Molecular Mechanisms That Confer Antibacterial Drug Resistance,” Nature, Vol. 406, No. 6797, 2000, pp. 775-781. http://dx.doi.org/10.1038/35021219

[3] A. Ashnagar and N. G. Naseri, "Analysis of Three Penicillin Antibiotics (Ampicillin, Amoxicillin and Cloxacillin) of Several Iranian Pharmaceutical Companies by HPLC,” Journal of Chemistry, Vol. 4, No. 4, 2007, pp. 536-545.

[4] Å. Sullivan, C. Edlund and C. E. Nord, "Effect of Antimicrobial Agents on the Ecological Balance of Human Microflora,” The Lancet Infectious Diseases, Vol. 1, No. 2, 2001, pp. 101-114. http://dx.doi.org/10.1016/S1473-3099(01)00066-4

[5] C. M. Kaye, A. Allen, S. Perry, M. McDonagh, M. Davy, K. Storm, N. Bird and O. Dewit, "The Clinical Pharmacokinetics of a New Pharmacokinetically Enhanced Formulation of Amoxicillin/Clavulanate," Clinical Therapeutics, Vol. 23, No. 4, 2001, pp. 578-584. http://dx.doi.org/10.1016/S0149-2918(01)80061-8

[6] S. M. Drawz and R. A. Bonomo, "Three Decades of $\beta$ Lactamase Inhibitors,” Clinical Microbiology Reviews, 
Vol. 23, No. 1, 2010, pp. 160-201. http://dx.doi.org/10.1128/CMR.00037-09

[7] M. Coll, J. Frau, B. Vilanova, J. Donoso, F. Munoz and F. G. Blanco, "Theoretical Study of the Alkaline Hydrolysis of a Bicyclic Aza- $\beta$-Lactam,” The Journal of Physical Chemistry B, Vol. 104, No. 47, 2000, pp. 11389-11394. http://dx.doi.org/10.1021/jp001989e

[8] World Health Organisation (WHO), "Quality Assurance of Pharmaceuticals: A Compendium of Guidelines and Related Materials,” Vol. 2, Geneva, 2004.

[9] S. K. Opuni, “Alert: Osons Chemists, Roxin Ghana Import Fake Medicines from Ghana,” My Joy Online, 2013. http://www.modernghana.com/news/445411/1/alert-osons -chemists-roxin-ghana-import-fake-medic.html

[10] S. Ayim, "List of Hospitals and Pharmacies in the Central Region of Ghana as recorded by the Regional Health Directorate,” Personal Interview, 2012.

[11] World Health Organisation (WHO), “The International Pharmacopoeia,” 4th Edition, 2nd Supplement, Geneva, 2008.

[12] Clinical and Laboratory Standards Institute, "Performance Standards for Antimicrobial Disk Susceptibility Test; Approved Standard," CLSI Document M02-A10, 10th ed. 2009.

[13] British Pharmacopoeia Commission, "British Pharmacopoeia 2013,” Renouf Publishing Company Limited, UK, 2013.

[14] S. Sengaloundeth, M. D Green, F. M. Fernández, Ot. Manolin, K. Phommavong, V. Insixiengmay, C. Y. Hampton, L. Nyadong, D. C. Mildenhall, D. Hostetler, L. Khounsaknalath, L. Vongsack, S. Phompida, V. Vanisaveth, L. Syhakhang and P. N. Newton, "A Stratified Random Survey of the Proportion of Poor Quality Oral Artesunate Sold at Medicine Outlets in the Lao PDR-Implications for Therapeutic Failure and Drug Resistance," Malaria Journal, Vol. 8, 2009, p. 172. http://dx.doi.org/10.1186/1475-2875-8-172

[15] P. N. Newton, M. D. Green, D. C. Mildenhall, A. Plançon, H. Nettey, L. Nyadong, D. M. Hostetler, I. Swamidoss, G. A. Harris, K. Powell, A. E. Timmermans, A. A. Amin, S. K. Opuni, S. Barbereau, C. Faurant, R. C. W. Soong, K. Faure, J. Thevanayagam, P. Fernandes, H. Kaur, B. Angus, K. Stepniewska, P. J. Guerin and F. M. Fernández, "Poor Quality Vital Anti-Malarials in Africa-An Urgent Neglected Public Health Priority,” Malaria Journal, Vol. 10, 2011, p. 352. http://dx.doi.org/10.1186/1475-2875-10-352

[16] E. A. Nyarko and H. Nettey, “Quality Assessment of Artemether/Lumefantrine Tablets Sampled from Pharmacies in Accra, Using the MVHimagePCv8.exe Color Software,” Pharmacology \& Pharmacy, Vol. 4, 2013, pp. 567-572. http://dx.doi.org/10.4236/pp.2013.48081

[17] J. Feng, X. Zhana, S. Qiaob, C. Wub and L. Xiaob, “A Mathematical Model for Calculating the Shelf Life of Ascorbic Acid Solution under Given Conditions,” Drug Development and Industrial Pharmacy, Vol. 38, No. 3, 2012, pp. 264-270.

http://dx.doi.org/10.3109/03639045.2011.598537

[18] D. Q. Craig, “The Mechanisms of Drug Release from Solid Dispersions in Water-Soluble Polymers," International Journal of Pharmaceutics, Vol. 231, No. 2, 2002, pp. 131-144. http://dx.doi.org/10.1016/S0378-5173(01)00891-2

[19] S. A. Qureshi and J. Shabnam, "Cause of High Variability in Drug Dissolution Testing and Its Impact on Setting Tolerances," European Journal of Pharmaceutical Sciences, Vol. 12, No. 3, 2001, pp. 271-276. http://dx.doi.org/10.1016/S0928-0987(00)00174-3 